\title{
Nuclear survivin in pN2 nonsmall cell lung cancer: prognostic and clinical implications
}

\author{
S. Mohamed*, , K. Yasufuku*, T. Nakajima*, K. Hiroshima", M. Chiyo*, \\ S. Yoshida*, M. Suzuki*, Y. Sekine*, K. Shibuya*, G. Agamy\#, \\ H. El-Shahhat" ${ }^{\#}$ T. Fujisawa* and I. Yoshino*
}

ABSTRACT: Patients with N2 nonsmall cell lung cancer (N2-NSCLC) represent heterogeneous groups. Survivin is a member of the inhibitor of apoptosis family. If N2-NSCLC patients could be stratified, based on survivin expression and/or its relation to cell cycle proteins, into homogeneous subgroups, certain therapies could be selected for those patients.

Survivin expression in 78 surgically resected primary pathological N2-NSCLC tumours was evaluated using immunohistochemistry. Relationships of survivin expression to overall survival, clinical features and expression of six cell cycle-related proteins (pRb, cyclin D1, p16 ${ }^{\text {INK4A }}$, p53, p21 ${ }^{\text {Waf1 }}$ and $\left.\mathrm{Ki}-67\right)$ were analysed.

Nuclear survivin and the number of mediastinal lymph node (LN) stations were independent prognostic factors. The patient group with combined negative survivin/single mediastinal LN station were the most favourable prognostic group, and was related to the clinical nodal factor. Indeed, patients with negative survivin/low Ki-67 labelling indices had the best survival, especially in nonsquamous histopathology.

The current authors conclude that nuclear survivin is strongly related to lymph node metastasis and proliferative potentials in pathological N2 nonsmall cell lung cancer patients. Pre-operative N2 nonsmall cell lung cancer patients with combined negative nuclear survivin and a single mediastinal lymph node station, or low proliferative indices, particularly in clinical N0-1 disease and nonsquamous histopathology, respectively, are expected to have a favourable post-operative prognosis and may be candidates for primary resection.

KEYWORDS: Nonsmall cell lung cancer, nuclear, pathological N2, prognosis, survivin

ung cancer is the most common cause of cancer-related mortality. Although surgical therapy remains the primary treatment for resectable disease, the composite 5-yr survival is only $16 \%$ [1]. In particular, patients with stage IIIA-N2 nonsmall cell lung cancer (NSCLC) represent incredibly heterogeneous groups, with regard to both prognosis and treatment $[2,3]$. Attempts to define homogeneous subgroups of N2-NSCLC patients have identified some prognostic factors $[3,4]$.

Nevertheless, if molecularly based factors that can be used as criteria for deciding whether to conduct surgery in patients with clinical N2NSCLC could be identified, the outcome of these patients could be improved [4].

Survivin is a $16.5-\mathrm{kDa}$ protein that is overexpressed in almost all malignancies, but rarely detected in normal differentiated adult tissues [5]. Functionally, survivin has been shown to inhibit apoptosis, regulate cell division and enhance angiogenesis $[5,6]$. Deletion of survivin resulted in a catastrophic defect of microtubule assembly, with absence of mitotic spindles, disorganised tubulin aggregates and multinucleation in the survivin knockout mice [7]. Nucleocytoplasmic localisation of survivin in tumour cells, determined by immunohistochemistry, has been reported to provide prognostic information in several types of cancer [8]. However, the prognostic significance of nuclear survivin expression remains controversial in different tumour types [9], including NSCLC [10, 11].

In a previous study, pathological (p)N2-NSCLC patients were classified into homogeneous prognostic subgroups, based on the expression of six cell cycle-related markers ( $p R b$, cyclin D1, p16 ${ }^{\text {INK4A }}, p 53, p 21^{\text {Waf1 }}$ and Ki-67) [4]. In the present study, the expression of both nuclear and cytoplasmic survivin was evaluated immunohistochemically in pN2-NSCLC patients, and the relationship of this expression to overall survival
AFFILIATIONS

Depts of *Thoracic Surgery, and

"Diagnostic Pathology, Graduate

School of Medicine, Chiba

University, Chiba, Japan.

\#Dept of Pulmonology, Faculty of Medicine, Assiut University, Assiut, Egypt.

\section{CORRESPONDENCE}

K. Yasufuku

Dept of Thoracic Surgery

Graduate School of Medicine

Chiba University

1-8-1 Inohana

Chuo-Ku

Chiba 260-8670

Japan

Fax: 81432262172

E-mail: kyasufuku@

faculty.chiba-u.jp

Received:

May 032008

Accepted after revision:

August 012008

\section{SUPPORT STATEMENT}

This study received grants-in-aid for scientific research from the Ministry of Education, Culture, Sports, Science and Technology of Japan, No. 19591611 to K. Yasufuku and No. 18790986 to T. Nakajima.

STATEMENT OF INTEREST

None declared. 
and clinical features was investigated. Moreover, the current authors searched for possible relationship(s) between survivin and cell cycle protein expression in N2-NSCLC patients. They hypothesised that N2-NSCLC patients could be stratified, based on survivin expression and/or its relation to cell cycle proteins, into homogeneous subgroups, for whom certain therapeutic strategies could be selected.

\section{MATERIALS AND METHODS}

\section{Study design}

To accomplish the aims of the current study, an immunohistochemical (IHC) analysis for survivin expression in primary surgically resected specimens of patients with pN2-NSCLC was performed. Following this, the prognostic implications of survivin expression with regard to overall survival and clinical features of those patients was studied. Lastly, the current authors searched for possible relationhip(s) between survivin expression and that of six cell cycle-related proteins [4] in 61 out of 78 patients.

\section{Patients and tissue samples}

Archived tissue blocks from 1990 to 1996 of surgically resected primary tumours with histologically proven N2-NSCLC (pN2NSCLC) were retrieved from the files of the Dept of Thoracic Surgery, Chiba University (Chiba, Japan). The study comprised 78 patients who had undergone surgical resection with curative intent. They were 58 males and 20 females with a mean age of 62.8 yrs. Histopathological diagnosis was carried out according to the World Health Organization specifications [12]. The tumours included 51 adenocarcinomas, 23 squamous cell carcinomas and four large cell carcinomas. All patients received neither induction chemotherapy nor pre-operative radiotherapy. Patients were excluded if they had metastatic NSCLC, a prior history of metastatic malignancy or neuroendocrine tumours. In total, 62 patients underwent lobectomy, 14 patients underwent pneumonectomy and two patients underwent segmentectomy. Deaths caused by operative complications were excluded. Primary tumours were staged as T1, T2 and T3 in 20, 38 and 20 patients, respectively.

Pre-operative staging included a computed tomography (CT) scan of the chest, a CT scan of the abdomen, fibreoptic bronchoscopy, brain magnetic resonance imaging and bone scintigraphy for all patients. Abdominal ultrasound was obtained only from patients who had an abnormality on their abdominal CT. Mediastinal lymph nodes (LNs) with a shortaxis dimension $\geqslant 1 \mathrm{~cm}$ on a CT scan were considered abnormal. All patients underwent mediastinal LN dissection at the time of surgery. Complete resection was defined as resection of all macroscopic tumour with the resection margins free of tumour on microscopic analysis. All patients had positive ipsilateral mediastinal LNs according to histological examination ( $\mathrm{pN} 2$ ). The tumours were classified according to the Japan Lung Cancer Society (Chiba, Japan) [13]. The classification scheme is identical to that of the International Union Against Cancer (Geneva, Switzerland). Some patients had clinically positive hilar (cN1) and mediastinal (cN2) LNs, defined as LNs that measured $\geqslant 1 \mathrm{~cm}$ on a CT scan before surgery. Out of 78 patients, 38 had clinical N2 (cN2) disease identified and, at the time of surgery, 31 out of 78 patients had multiple-station enlargement of mediastinal (N2) LNs identified.
All patients were followed up for $10 \mathrm{yrs}$, and the mean survival was 3.46 yrs. Out of the 78 patients, 55 died, and deaths were attributable to cancer in $44(80.0 \%)$ out of 55 patients. The study was approved by the Chiba University Institutional Review Board (approval No. 119).

\section{Immunohistochemistry}

IHC analysis of the specimens for the expression of survivin (nuclear and cytoplasmic) in $78 \mathrm{pN} 2-$ NSCLC patients was performed. In addition, 61 out of 78 patients were evaluated for the expression of six cell cycle-related proteins ( $\mathrm{pRb}$, cyclin $\mathrm{D} 1, \mathrm{p} 16^{\mathrm{INK} 4 \mathrm{~A}}, \mathrm{p} 53, \mathrm{p} 21^{\text {Waf1 }}$ and Ki-67), as previously described [4]. For survivin immunostaining, the IHC assay was carried out on $10 \%$ formalin-fixed, paraffin-embedded tissue sections that were cut to a thickness of 3 or $4 \mu \mathrm{m}$ and mounted on glass slides. All sections were then dewaxed in xylene, rehydrated through a graded alcohol series and washed in Tris-buffered saline with Tween 20 (DakoCytomation, Carpinteria, CA, USA). This buffer was used for all subsequent washes and for dilution of the antibodies. Antigen retrieval was achieved via heating in an autoclave at $121^{\circ} \mathrm{C}$ for $15 \mathrm{~min}$, after immersion of the tissue slides into target retrieval solution (DakoCytomation). Following this, all tissue sections were processed with the DAKO catalysed signal amplification horse radish peroxidase system (code K1500; DakoCytomation). Mouse monoclonal antibody raised against full-length recombinant survivin (DakoCytomation) was used at dilutions of 1:400. The primary antibody was incubated overnight at $4^{\circ} \mathrm{C}$. As the final chromogen, 3,3'-diaminobenzidine was used, and haematoxylin was used as the nuclear counterstain. Positive tissue controls were included in each experiment and consisted of tissues that had previously been stained specifically for the target antigen after exposure to primary antibody. Each slide was examined independently by two observers (S. Mohamed and K. Hiroshima) without knowledge of the patients' clinical data. Cytoplasmic survivin immunoreactivity was evaluated semiquantitatively based on the intensity of staining [11] and was scored as weak $(+1)$, moderate $(+2)$ or intense $(+3)$. In tumours with heterogeneous immunostaining, the predominant pattern was considered for scoring. Specimens with no or weak staining were considered negative, whereas those with moderate and intense staining were considered positive [11]. For nuclear survivin, immunoreactivity was considered positive when $>10 \%$ of tumour nuclei were positively stained [14].

\section{Statistical analysis}

The associations between IHC parameters, as well as IHC/ clinico-pathological ones, were analysed by the use of the Chisquared test and/or Fisher exact test. The IHC parameters were: survivin, both nuclear and cytoplasmic; and cell cyclerelated proteins [4]. The clinico-pathological patient parameters included: age, sex, histopathological type, pathological tumour status, clinical nodal $(\mathrm{cN})$ status, and the number of mediastinal LN stations involved. A univariate survival analysis of each prognostic variable was used to estimate overall survival according to the Kaplan-Meier method [15]. The prognostic variables for overall survival included the abovementioned clinico-pathological parameters, as well as nuclear and cytoplasmic survivin. Overall survival was calculated from the date of surgery to the date of either death or the last followup. The terminal event was death attributable to cancer or 

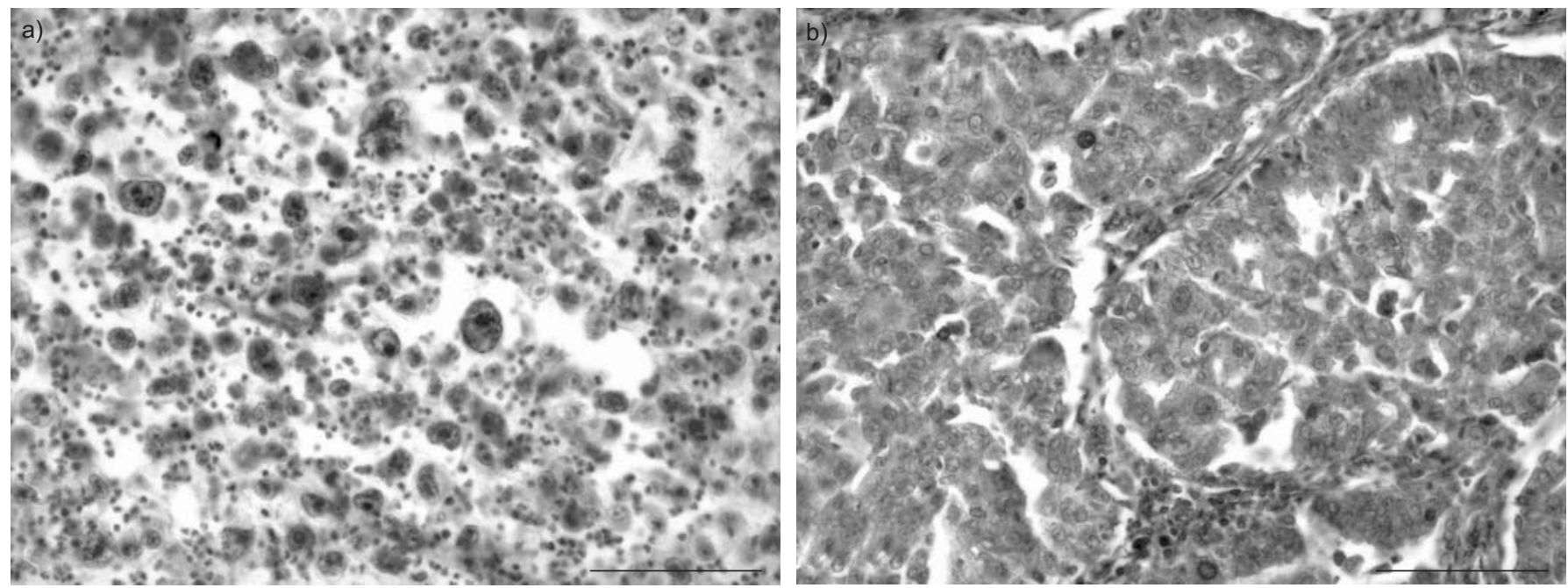

FIGURE 1. Immunoreactivity patterns of survivin expression in a representative adenocarcinoma specimen of pathological N2 nonsmall cell lung cancer, showing a) positively stained nuclei of neoplastic cells (nuclear survivin) and b) intense cytoplasmic staining of malignant cells (cytoplasmic survivin). Scale bars $=100 \mu \mathrm{m}$.

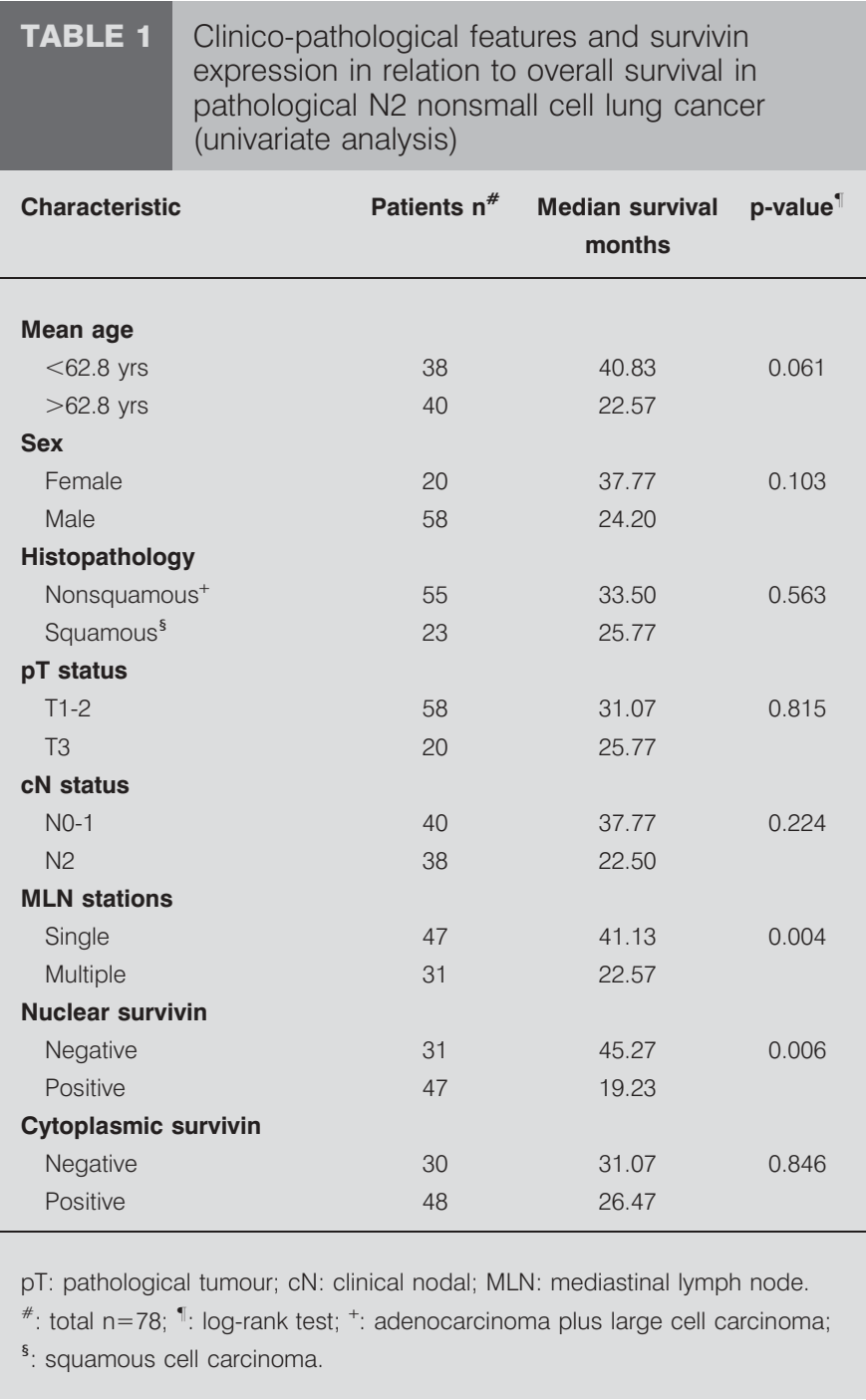

noncancer causes. The significance of the differences in survival distribution among prognostic groups was evaluated with the log-rank test. A Cox proportional hazards model was applied to the multivariate survival analysis [16]. The criterion of significance chosen was $\mathrm{p}<0.05$, and all tests were two-tailed.

\section{RESULTS}

\section{Nuclear and cytoplasmic survivin immunoreactivity in pN2-NSCLC}

Survivin immunoreactivity was detected in 68 (87.2\%) out of 78 tumours examined (fig. 1). Nuclear immunoreactivity was observed in $47(60.3 \%)$ out of 78 cases (fig. 1a). Cytoplasmic immunoreactivity was detected in $48(61.5 \%)$ out of 78 tumours, with an intensity that was usually homogeneous and uniform within each case (fig. 1b). Indeed, 27 (34.6\%) tumours showed both cytoplasmic and nuclear immunoreactivity and in $10(12.8 \%)$ cases neither cytoplasmic nor nuclear immunostaining was observed. Interestingly, there was no significant relationship between nuclear and cytoplasmic survivin expression $(p=0.367)$.

\section{Clinico-pathological features and survivin expression in relation to overall survival}

The 5-yr survival rate for the included patients was $25.6 \%$. Univariate survival analysis revealed that only the number of mediastinal LN stations and nuclear survivin expression significantly influenced survival (table 1).

Patients with multiple and single mediastinal nodal stations had a median survival time of 22.57 and 41.13 months, respectively ( $p=0.004$; fig. 2 ). Moreover, patients with nuclear survivin overexpression had poorer survival than those with negative expression, with a median survival of 19.23 and 45.27 months, respectively ( $p=0.006$; fig. 3 ). According to the results of univariate analysis, multivariate analysis was performed and indicated that the number of mediastinal LN stations and nuclear survivin expression were the independent prognostic factors in this series of $\mathrm{pN} 2-\mathrm{NSCLC}$ patients. The 


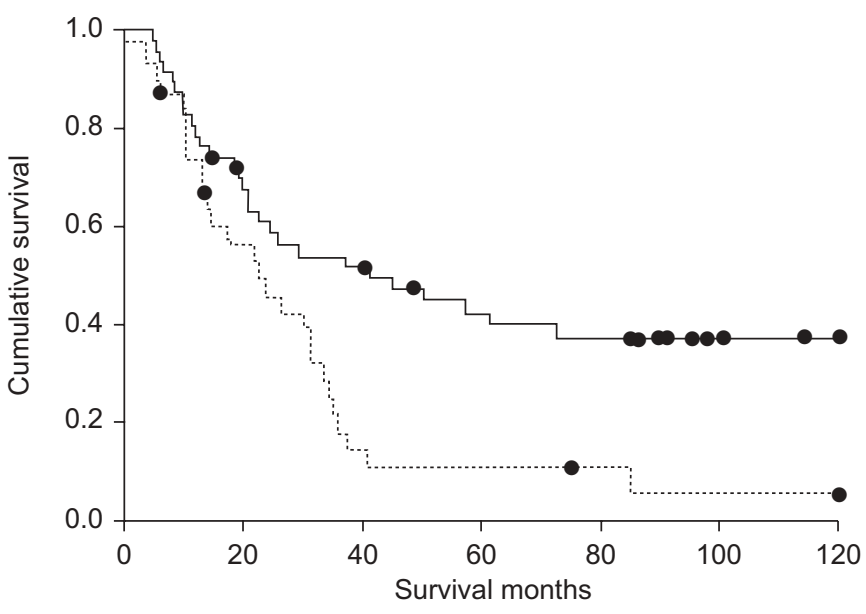

FIGURE 2. Kaplan-Meier survival curves in patients with pathological N2 nonsmall cell lung cancer show that multi-station mediastinal lymph node (MLN) involvement is a bad prognostic factor ( $p=0.004$ for comparison of the two curves). —: single-station MLN, $n=47 ; \cdots$ : multi-station $M L N, n=31$. $\bullet$ : number of survivors in each group.

calculated relative risk of death for patients with multiple nodal stations was 2.232 (95\% confidence interval $0.260-0.772$; $\mathrm{p}=0.004)$, and that for patients with nuclear survivin overexpression was $2.208((0.257-0.797) ; \mathrm{p}=0.006$; table 2$)$.

\section{Immunohistochemical-clinical combinations}

Based on the results of multivariate survival analysis, the patients with pN2-NSCLC were divided into four groups, as follows. Group A: survivin negative, single station $(n=19)$; group B: survivin negative, multiple stations $(n=12)$; group C: survivin positive, single station $(n=28)$; and group D: survivin positive, multiple stations $(n=19)$.

Survival analysis of these four combinations revealed that group A patients had the most favourable overall survival, with a median survival time of 61.23 months, compared with that of group D patients, with a median survival time of 14.57 months $(p=0.0008)$. Morerover, when the possible relationship(s) between these combinations and the clinicopathological patient features were examined, an interesting relationship was found for the $\mathrm{cN}$ status: $16(84.2 \%)$ out of 19 patients in group A had cN0-1 stage, whereas three $(15.8 \%)$ out of 19 patients had $\mathrm{cN} 2(\mathrm{p}=0.040$; table 3$)$.

\section{Relationship between survivin and cell cycle proteins}

The current authors searched for the possible relationship(s) between survivin and six cell cycle proteins among 61 out of 78 patients with pN2-NSCLC. Among this panel of six cell cyclerelated markers, only the Ki-67 labelling index (LI) was significantly related to nuclear survivin expression. In total, $28(77.8 \%)$ out of 36 cases with high Ki-67 LI had concurrent survivin overexpression, compared with eight (22.2\%) out of 36 cases with high Ki-67 LI that had negative survivin expression $(p=0.003)$. Conversely, no significant relationship was found between cytoplasmic survivin expression and that of any of the six cell cycle markers. Also, based on this survivin/Ki-67 association, pN2-NSCLC patients were divided into four prognostic groups, as follows. Group 1: survivin

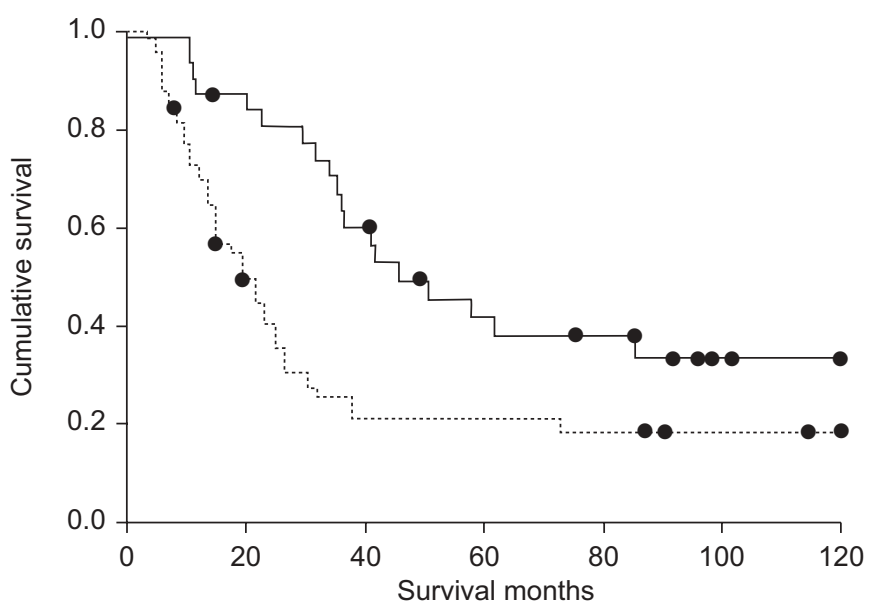

FIGURE 3. N2 nonsmall cell lung cancer patients with nuclear survivin overexpression $(\cdots ; n=47)$ have significantly lower overall survival than those with negative expression ( $-; n=31 ; p=0.006$ ). $\bullet$ : number of survivors in each group.

negative, low Ki-67 LI ( $\mathrm{n}=15)$; group 2: survivin negative, high Ki-67 LI ( $n=8)$; group 3: survivin positive, low Ki-67 LI $(n=10)$; and group 4: survivin positive, high Ki-67 LI $(n=28)$. Again, survival analysis of these combinations revealed that group 1 patients had the most favourable overall survival, compared with that of group 4 patients, with median survival times of 50.53 and 14.17 months, respectively $(p=0.009)$. Finally, a significant association was found between these combinations and the histopathological types: 13 (86.7\%) and two (13.3\%) out of 15 patients in group 1 had nonsquamous and squamous histopathology, respectively $(p=0.027$; table 4$)$.

\section{DISCUSSION}

The current study results revealed that nuclear survivin as well as the number of affected mediastinal LN stations are independent prognostic factors in patients with N2-NSCLC. Moreover, nuclear survivin and its relations to the nodal factor and proliferative activity in those patients might help their selection into certain therapeutic strategies.

The burden of disease, hence the prognosis, in stage IIIA-N2 NSCLC varies from microscopic, single-station, mediastinal nodal involvement to bulky, multi-station, fixed, mediastinal

TABLE 2 Multivariate Cox regression analysis of overall survival in pathological N2 nonsmall cell lung cancer patients

\begin{tabular}{lccc} 
Characteristic & RR of death & $\begin{array}{c}\text { 95\% confidence } \\
\text { interval }\end{array}$ & p-value \\
\hline $\begin{array}{l}\text { MLN stations } \\
\text { Single } \\
\text { Multiple }\end{array}$ & 1 & & 0.004 \\
$\begin{array}{l}\text { Nuclear survivin } \\
\text { Negative }\end{array}$ & 2.232 & $0.260-0.772$ & 0.006 \\
Positive & 1 & & \\
\hline
\end{tabular}

RR: relative risk; MLN: mediastinal lymph node 
TABLE 3 Nuclear survivin and mediastinal lymph node station combinations in relation to survival and clinical nodal (cN) status

\begin{tabular}{|c|c|c|c|c|c|c|}
\hline \multirow[t]{2}{*}{ Group } & \multirow{2}{*}{$\begin{array}{c}\text { Patients } \\
\mathrm{n}^{\#}\end{array}$} & \multirow{2}{*}{$\begin{array}{l}\text { Median survival } \\
\text { months }\end{array}$} & \multirow[t]{2}{*}{ p-value } & \multicolumn{3}{|c|}{$\mathrm{cN}$ status } \\
\hline & & & & $\mathrm{cNO}-1$ & $\mathrm{cN} 2$ & p-value ${ }^{+}$ \\
\hline A: Survivin negative, single station & 19 & 61.23 & 0.0008 & 16 & 3 & 0.040 \\
\hline B: Survivin negative, multiple stations & 12 & 34.80 & & 4 & 8 & \\
\hline C: Survivin positive, single station & 28 & 20.73 & & 11 & 17 & \\
\hline
\end{tabular}

nodal disease $[2,3]$. While there is consensus to treat patients with bulky-N2 in the same group as locally advanced IIIB disease, and to treat with primary surgical resection patients with incidental or minimal N2 involvement, still there is no agreement about the best approach to patients with ipsilateral mediastinal LN metastasis diagnosed pre-operatively, although considered technically potentially resectable [17]. Furthermore, studies comparing surgical intervention alone with pre-operative chemotherapy followed by surgical resection, for patients with clinically evident N2 disease, have shown conflicting results [18]. Molecularly based stratification of N2-NSCLC patients into homogeneous subgroups can help selection of those patients who might benefit from certain therapeutic strategies, thus improving their outcome [4]. The rationale for investigating survivin as a prognostic marker in malignancy is based on its ability to inhibit apoptosis, promote proliferation and enhance angiogenesis $[5,6,8]$. Owing to its involvement in these processes, survivin is likely to be causally involved in tumour progression and, consequently, increased levels would be expected to predict aggressive disease [5]. Indeed, several reports have shown that high tumour levels of survivin are associated with adverse outcome in patients with different types of cancer $[5,6]$, including NSCLC $[10,11]$.

The current results revealed that the majority $(87.2 \%)$ of tumour samples showed survivin immunoreactivity and survival analysis could reveal important relevances. Univariate survival analysis showed that only the number of affected mediastinal LN stations and nuclear survivin affected survival. Moreover, multivariate analysis revealed that those two factors were independent prognostic factors for N2-NSCLC patients. The socalled N2-bulky (multi-station) disease is a factor well known to be associated with an inverse prognostic outcome in N2-NSCLC patients $[2,3]$.

Due to the large difference in expression between cancer and corresponding normal tissue, and being a multifunctional protein that plays vital roles in various cancer-related aspects, it is not surprising that survivin expression had prognostic significance in various cancer types [5]. The present results confirm this prognostic significance of survivin in NSCLC [5, 11]. Conversely, the prognostic relevance of the subcellular pools of survivin has been a matter of debate in many cancers [9], including NSCLC [10, 11]. These controversies could be explained, in IHC studies, on the bases of using antibodies of different specificities or concentrations, and employing different cut-off points and different approaches for storing and processing tissues [5, 9]. In this regard, the current data revealed that nuclear survivin can be utilised as a prognostic marker for N2-NSCLC. However, the data also confirm that cytoplasmic survivin expression had neither biological nor clinical value in the current N2-NSCLC series. Combining these two prognostic factors, it was possible to stratify pN2NSCLC patients into prognostic subgroups. Patients with combined single-station mediastinal LN involvement and negative nuclear survivin had the best overall survival. Indeed, these combinations were significantly related to the $\mathrm{cN}$ status of pN2-NSCLC patients. The current results are consistent with those that reported that survivin was a marker of LN metastasis $[19,20]$. The link between survivin expression and the potential for LN metastasis could be explained in two ways. First, survivin could be an apoptosis inhibitor: the proportion of cancerous cells in a tissue, which would otherwise be removed by apoptosis, increases with continued

TABLE 4 Nuclear survivin and Ki-67 labelling index combinations in relation to survival and histopathology

\begin{tabular}{|c|c|c|c|c|c|c|}
\hline \multirow[t]{2}{*}{ Group } & \multirow{2}{*}{$\begin{array}{c}\text { Patients } \\
\mathrm{n}^{\#}\end{array}$} & \multirow{2}{*}{$\begin{array}{l}\text { Median survival } \\
\text { months }\end{array}$} & \multirow[t]{2}{*}{$p$-value } & \multicolumn{3}{|c|}{ Histopathology } \\
\hline & & & & Nonsquamous $^{+}$ & Squamous ${ }^{\S}$ & p-value ${ }^{f}$ \\
\hline 1: Survivin negative, low Ki-67 & 15 & 50.53 & 0.009 & 13 & 2 & 0.027 \\
\hline 2: Survivin negative, high Ki-67 & 8 & 29.03 & & 4 & 4 & \\
\hline 3: Survivin positive, low Ki-67 & 10 & 37.13 & & 9 & 1 & \\
\hline 4: Survivin positive, high Ki-67 & 28 & 14.17 & & 15 & 13 & \\
\hline
\end{tabular}

\#: total $\mathrm{n}=61 ;{ }^{\bullet}$ : log-rank test; ${ }^{+}$: adenocarcinoma plus large cell carcinoma; ${ }^{\S}$ : squamous cell carcinoma; ${ }^{f}$ : Fisher's exact test. 
growth and increased potential for invasion and metastasis [19]. Secondly, survivin could be related to microvessel density and enhanced angiogenesis [6], both of which are strongly associated with a high potential for LN metastasis. Taking into consideration that metastasis to the ipsilateral mediastinal LNs (N2 disease) is the most important prognostic factor in completely resectable NSCLC [2, 21], together with this relevance of survivin to LN metastasis, the present results could have important implications for pre-operative planning of N2-NSCLC patients. Thus, patients with combined singlestation mediastinal LN and negative nuclear survivin, especially those with clinical N0-1 stage, are expected to have favourable post-operative prognosis and could be candidates for primary resection.

Finding a possible relationship between survivin expression and that of the cell cycle-related proteins would be of interest. Therefore, the current authors examined the evidence for such a relationship in 61 out of 78 patients with pN2-NSCLC. Among a panel of six cell cycle-related markers [4], nuclear survivin was related only to Ki-67 (proliferative) LI. This finding is in agreement with many studies that have reported a strong link between survivin expression and increased tumour proliferative activivity [5-7, 22]. Survivin has been implicated in a dual role connecting suppression of apoptosis to regulation of chromosomal segregation and cell division [8]. Targeting experiments using antisense survivin or dominantnegative mutants resulted in spontaneous apoptosis, increased caspase activity and inhibition of cell proliferation [23]. The protective anti-apoptotic effect of survivin in proliferating malignant cells may be a mechanism for stabilising tumour cells with chromosomal abnormalities, favouring the survival of these cells and the progression of the tumours [7]. Furthermore, IKEGUCHI et al. [22] concluded that survivin gene expression may control cell proliferation rather than apoptosis in oesophageal cancer. Remarkably, the current data support the fact that, in genetically normal proliferating cells, Ki-67 and survivin should be completely linearly related on the basis that $\mathrm{Ki}-67$ is present for $75 \%$ of the duration of the S-phase and survivin only for a 2-3-h period of G2-M [24]. However, although $\mathrm{Ki}-67$ is not overexpressed as an oncogene, it could be that survivin is, thus justifying a concomitant study of both factors. Whether uncoupling of both Ki-67 and survivin should be considered an aberrant proliferation needs to be further evaluated.

Similarly, it was possible to group N2-NSCLC patients into prognostic subsets, according to nuclear survivin/Ki-67 LI combinations. The patient group with concurrent low proliferative indices and negative nuclear survivin had the best overall survival. The demonstration that survivin-negative and low Ki-67 LI patients had the best survival might be expected and was adequately demonstrated in the present data. Interestingly, $86.7 \%$ of this group had nonsquamous histopathology. Both survivin overexpression and high Ki-67 LI were related to squamous histopathology in oesophageal cancer [25] and NSCLC [26], respectively. Thus, N2-NSCLC patients with concurrent negative nuclear survivin and low $\mathrm{Ki}$ 67 indices, particularly those with nonsquamous histopathology, are expected to have favourable post-operative prognosis and could be candidates for primary resection.
In addition, the clinical utility of the present results could be highlighted by the use of real-time endobronchial ultrasoundguided transbronchial needle aspiration (EBUS-TBNA) as a minimally invasive and accurate tool for pre-operative staging of NSCLC patients [27]. Indeed, it could be expected that preoperative evaluation of N2-NSCLC patients, using survivin and/or Ki-67 expression in EBUS-TBNA-obtained mediastinal nodal tissue samples, would be clinically useful [28, 29]. From a therapeutic point of view, survivin is a promising marker for anticancer therapy $[5,30]$ and it is anticipated to be of value for molecularly targeted therapy of N2-NSCLC in the near future. The present study could have one limitation, which is that it was retrospective. Therefore, further prospective studies evaluating molecular markers in N2-NSCLC are warranted.

\section{CONCLUSION}

The current results suggest that nuclear survivin and the number of affected mediastinal lymph node stations are two independent prognostic factors in pathological N2 nonsmall cell lung cancer. Nuclear survivin is strongly related to lymph node metastasis and proliferative potentials in N2 nonsmall cell lung cancer patients. Pre-operative N2 nonsmall cell lung cancer patients with combined negative nuclear survivin and a single mediastinal lymph node station or low proliferative indices, particularly in clinical N0-1 disease and nonsquamous histopathology, respectively, are expected to have a favourable post-operative prognosis and may be candidates for primary resection.

\section{ACKNOWLEDGEMENTS}

The authors would like to thank R. Kubo (Dept of Thoracic Surgery, Chiba University, Chiba, Japan) for her technical aid.

\section{REFERENCES}

1 American Cancer Society. Cancer Facts and Figures 2007. Atlanta, American Cancer Society, 2007; pp. 13-14.

2 Ruckdeschel JC. Combined modality therapy of non-small cell lung cancer. Semin Oncol 1997; 24: 429-439.

3 Keller SM, Vangel MG, Wagner H, et al. Prolonged survival in patients with resected non-small cell lung cancer and single-level N2 disease. J Thorac Cardiovasc Surg 2004; 128: 130-137.

4 Mohamed S, Yasufuku K, Hiroshima K, et al. Prognostic implications of cell cycle-related proteins in primary resectable pathologic N2 nonsmall cell lung cancer. Cancer 2007; 109: 2506-2514.

5 Duffy MJ, O'Donovan N, Brennan DJ, Gallagher WM, Ryan BM. Survivin: a promising tumor biomarker. Cancer Lett 2007; 249: 49-60.

6 Kawasaki H, Toyoda M, Shinohara H, et al. Expression of survivin correlates with apoptosis, proliferation, and angiogenesis during human colorectal tumorigenesis. Cancer 2001; 91: 2026-2032.

7 Uren AG, Wong L, Pakusch M, et al. Survivin and the inner centromere protein INCENP show similar cell-cycle localization and gene knockout phenotype. Curr Biol 2000; 10: 1319-1328.

8 Fortugno P, Wall NR, Giodini A, et al. Survivin exists in immunochemically distinct subcellular pools and is 
involved in spindle microtubule function. J Cell Sci 2002; 115: 575-585.

9 Li F, Yang J, Ramnath N, Javle MM, Tan D. Nuclear or cytoplasmic expression of survivin: what is the significance? Int J Cancer 2005; 114: 509-512.

10 Vischioni B, van der Valk P, Span SW, Kruyt FA, Rodriguez JA, Giaccone G. Nuclear localization of survivin is a positive prognostic factor for survival in advanced non-small-cell lung cancer. Ann Oncol 2004; 15: 1654-1660.

$11 \mathrm{Lu}$ B, Gonzalez A, Massion PP, et al. Nuclear survivin as a biomarker for non-small-cell lung cancer. Br J Cancer 2004; 91: 537-540.

12 Travis WD, Brambilla E, Müller-Hermelink HK, Harris CC, eds, World Health Organization Classification of Tumours. Pathology and Genetics of Tumours of the Lung, Pleura, Thymus and Heart. Lyon, International Agency for Research on Cancer, 2004; p 10.

13 The Japan Lung Cancer Society. Classification of Lung Cancer. 1st English Edn. Tokyo, Kanehara \& Co., Ltd, 2000; pp. 1-114.

14 Ikehara M, Oshita F, Kameda Y, et al. Expression of survivin correlated with vessel invasion is a marker of poor prognosis in small adenocarcinoma of the lung. Oncol Rep 2002; 9: 835-838.

15 Kaplan EL, Meier P. Nonparametric estimation from incomplete observations. J Am Stat Assoc 1958; 53: 457-481.

16 Cox DR. Regression models and life-tables. J R Stat Soc Ser B 1972; 34: 187-220.

17 De Marinis F, Gebbia V, De Petris L. Neoadjuvant chemotherapy for stage IIIA-N2 non-small cell lung cancer. Ann Oncol 2005; 16: Suppl. 4, iv116-iv122.

18 Nagai K, Tsuchiya $\mathrm{R}$, Mori $\mathrm{T}$, et al. A randomized trial comparing induction chemotherapy followed by surgery with surgery alone for patients with stage IIIA N2 nonsmall cell lung cancer (JCOG 9209). J Thorac Cardiovasc Surg 2003; 125: 254-260.

19 Miyachi K, Sasaki K, Onodera S, et al. Correlation between survivin mRNA expression and lymph node metastasis in gastric cancer. Gastric Cancer 2003; 6: 217-224.
20 Deng $\mathrm{H}, \mathrm{Wu}$ RL, Zhou HY, Huang X, Chen Y, Liu LJ. Significance of survivin and PTEN expression in full lymph node-examined gastric cancer. World J Gastroenterol 2006; 12: 1013-1017.

21 Martini N, Flehinger BJ. The role of surgery in N2 lung cancer. Surg Clin North Am 1987; 67: 1037-1049.

22 Ikeguchi M, Yamaguchi K, Kaibara N. Survivin gene expression positively correlates with proliferative activity of cancer cells in esophageal cancer. Tumour Biol 2003; 24: 40-45.

23 Olie RA, Simões-Wüst AP, Baumann B, et al. A novel antisense oligonucleotide targeting survivin expression induces apoptosis and sensitizes lung cancer cells to chemotherapy. Cancer Res 2000; 60: 2805-2809.

24 Mellai M, Caldera V, Patrucco A, Annovazzi L, Schiffer D. Survivin expression in glioblastomas correlates with proliferation, but not with apoptosis. Anticancer Res 2008; 28: 109-118.

25 Rosato A, Pivetta M, Parenti A, et al. Survivin in esophageal cancer: an accurate prognostic marker for squamous cell carcinoma but not adenocarcinoma. Int $J$ Cancer 2006; 119: 1717-1722.

26 Shiba M, Kohno H, Kakizawa K, et al. Ki-67 immunostaining and other prognostic factors including tobacco smoking in patients with resected nonsmall cell lung carcinoma. Cancer 2000; 89: 1457-1465.

27 Yasufuku K, Chiyo M, Koh E, et al. Endobronchial ultrasound guided transbronchial needle aspiration for staging of lung cancer. Lung Cancer 2005; 50: 347-354.

28 Nakajima T, Yasufuku K, Suzuki M, et al. Assessment of epidermal growth factor receptor mutation by endobronchial ultrasound-guided transbronchial needle aspiration. Chest 2007; 132: 597-602.

29 Mohamed S, Yasufuku K, Nakajima T, et al. Analysis of cell cycle-related proteins in mediastinal lymph nodes of patients with N2-NSCLC obtained by EBUS-TBNA: relevance to chemotherapy response. Thorax 2008; 63: 642-647.

30 Altieri DC. Validating survivin as a cancer therapeutic target. Nat Rev Cancer 2003; 3: 46-54. 